\title{
La radioprotection dans l'établissement de La Hague
}

\author{
C. LAFFAILLE $\left(^{*}\right)$ \\ (Manuscrit reçu le 11 août 1980)
}

\begin{abstract}
RÉSUMÉ
Après une description rapide du principe de fonctionnement de l'Usine de retraitement et des caractéristiques des combustibles, on décrit comment sont conduites les actions de sécurité dans l'établissement en fonction des risques rencontrés. Si la limitation des doses intégrées par le personnel est un objectif d'ores et déjà atteint, on pense que le seuil d'optimisation des doses n'est pas acquis et que d'autres approches sont possibles, qu'il convient de mettre en œuvre sans tarder.
\end{abstract}

\begin{abstract}
After a rapid description of the working rules of the reprocessing facility and the fuel characteristics, we describe how the safety actions are led in the plant with respect to the occasional risks. If the limitation of the doses integrated by the workers is an objective which has been reached, we think the optimal level is not secured and that other approaches are possible, which must be used without delay.
\end{abstract}

\section{PRINCIPE DE FONCTIONNEMENT DE L'USINE}

L'usine de La Hague conçue pour le retraitement des combustibles de la filière uranium naturel-graphite gaz (UNGG) a été en partie modifiée pour pouvoir aussi retraiter les combustibles de la filière à eau légère (EL).

Depuis 1976, le retraitement des combustibles oxydes EL s'effectue en alternance avec le retraitement des combustibles UNGG et croît donc relativement lentement du fait des campagnes de retraitement des combustibles UNGG qui restent importantes et prioritaires.

C'est ainsi que 240 t de combustibles EL ont été retraitées dans de bonnes conditions de sécurité ainsi que, pour mémoire, $2 \mathrm{t}$ de combustibles du réacteur à neutrons rapides Phenix.

(*) COGEMA, Centre de La Hague, Service de Prévention et Radioprotection, B.P. $\mathrm{n}^{\circ} 270,50107$ Cherbourg. 
Schéma du retraitement des combustibles Usine UP2 (HAO)

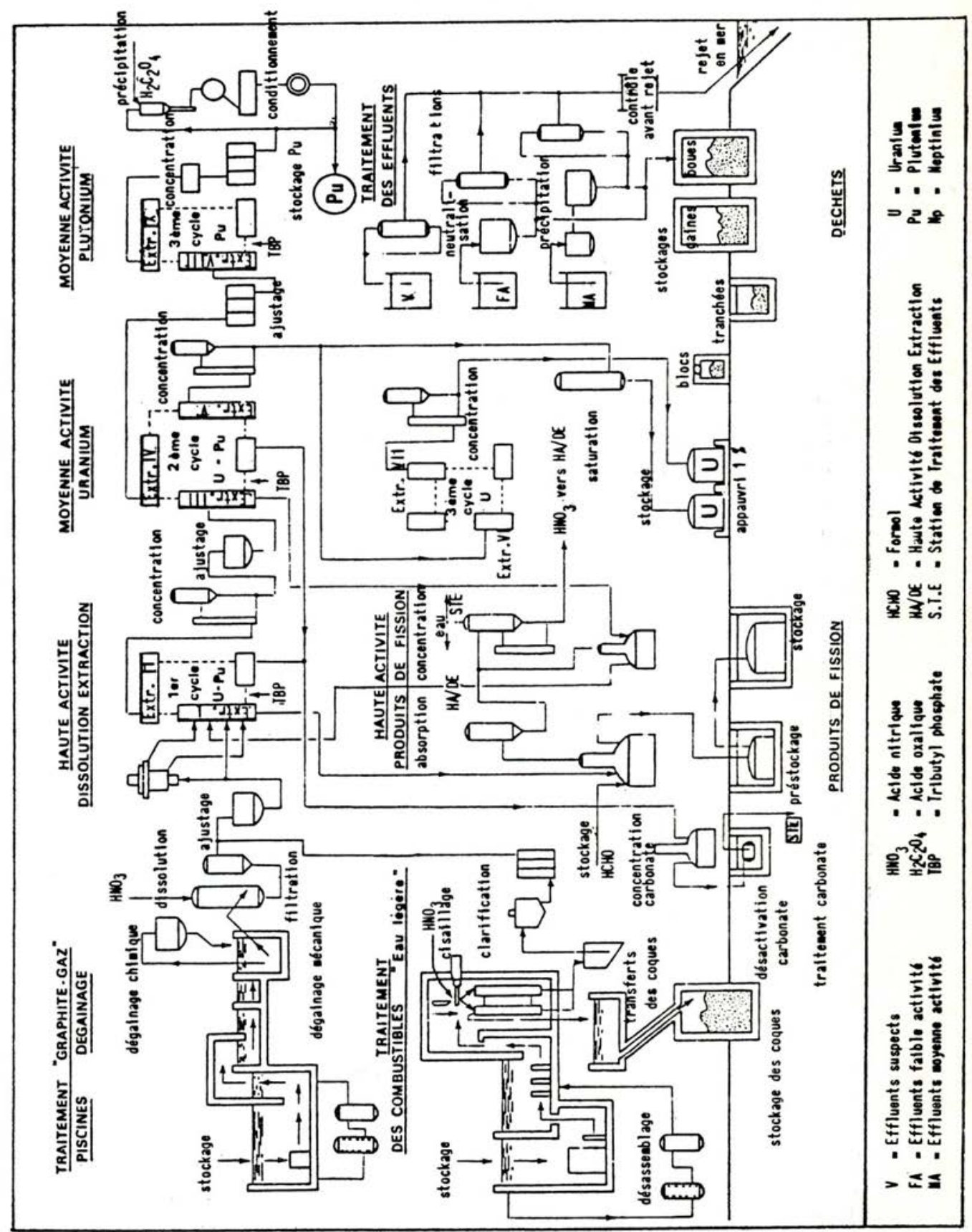

FIG. 1 
La figure 1 montre le principe de fonctionnement de l'usine, on y distingue 8 fonctions principales :

- réception et stockage des combustibles;

- préparation du combustible en vue de dissolution et dissolution avec lavage des gaz (ces deux premières fonctions sont effectuées dans des unités différentes selon qu'il s'agit de combustibles UNGG ou EL;

- premier cycle d'extraction permettant la séparation de l'uranium et du plutonium des produits de fission;

- concentration des produits de fission et stockage sous forme liquide (cette unité permet également de récupérer de l'acide nitrique et de l'eau qui seront recyclés dans le procédé);

- deuxième cycle d'extraction de l'uranium et du plutonium suivi d'une séparation de ces deux produits; en oxyde;

- ultime purification du plutonium en phase liquide, puis transformation

- purification, concentration et stockage de l'uranium;

- traitement des effluents liquides avant rejet en mer.

\section{CARACTÉRISTIQUES DES COMBUSTIBLES RETRAITÉS}

\subsection{CARACtÉRISTIQUeS PHYSIQUeS}

\section{Eau légère}

Nature du gainage : Zircalloy ou acier inoxydable.

Longueur : $4 \mathrm{~m}$ environ.

Embouts de pied et de tête : acier inoxydable.

Nature du combustible : $\mathrm{UO}_{2}$.

Masse de combustible : 100 à $500 \mathrm{~kg}$ selon le type d'élément combustible.

\section{$U N G G$}

Nature du gainage : magnésium.

Longueur : $60 \mathrm{~cm}$.

Nature du combustible : uranium métal allié.

Masse du combustible : 10 à $25 \mathrm{~kg}$ suivant le type d'élément combustible.

\subsection{Caractéristiques Radioactives}

Les caractéristiques nominales sont décrites au tableau I.

On remarque essentiellement, outre des caractéristiques physiques très différentes, que le retraitement des combustibles oxydes entraîne, par rapport au retraitement des combustibles UNGG et pour des durées de refroidissement différentes, pour une tonne de combustible retraité :

- une activité alpha dix fois plus importante due tant au plutonium qu'aux autres transplutoniens,

RADIOPROTECTION 


\section{TABLEAU I}

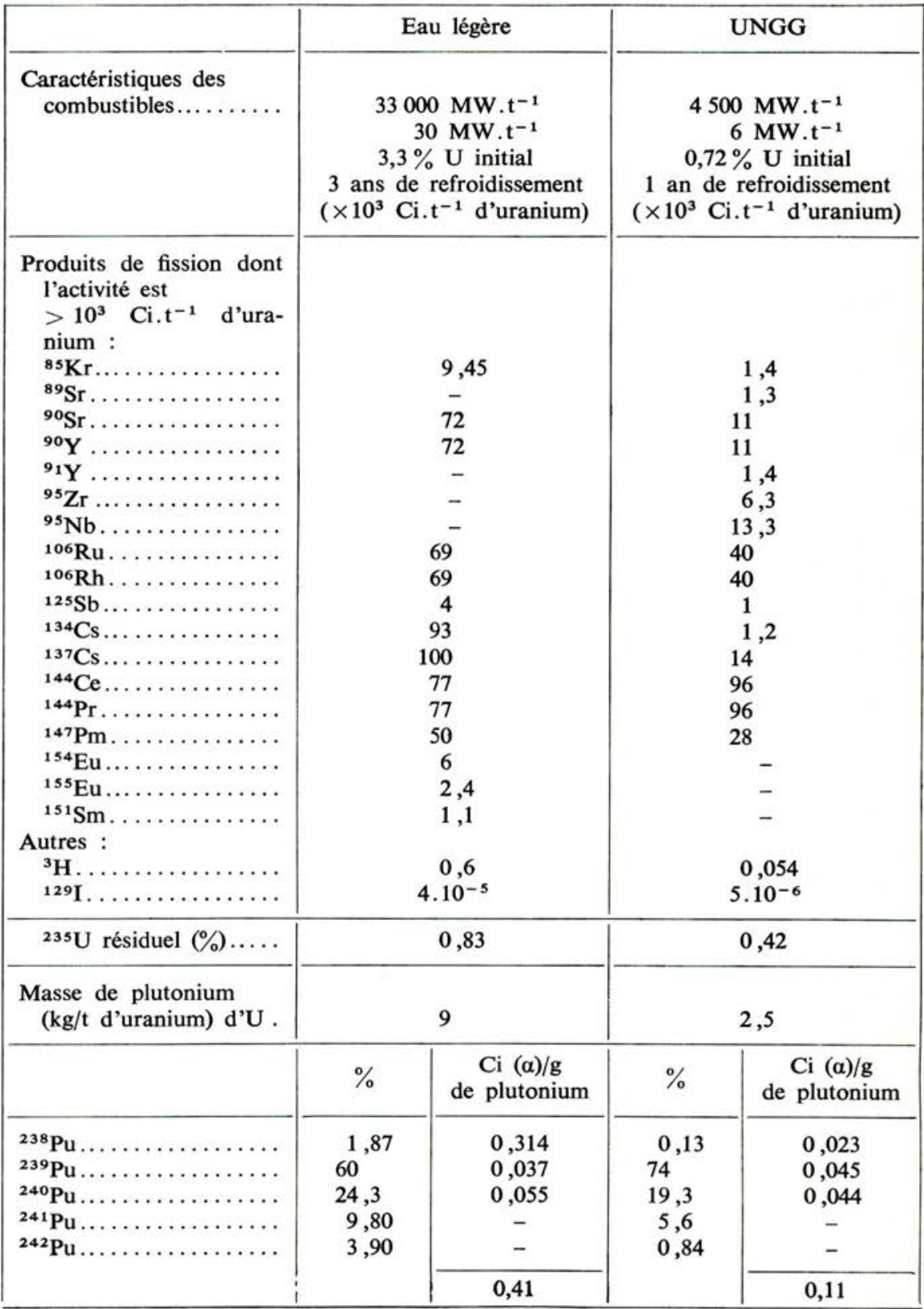


TABLEAU I (suite)

\begin{tabular}{|c|c|c|}
\hline 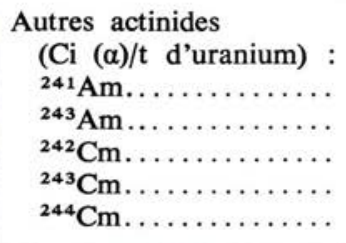 & $\begin{array}{r}619 \\
17 \\
271 \\
4 \\
2250\end{array}$ & $\begin{array}{c}38 \\
- \\
319 \\
- \\
8\end{array}$ \\
\hline $\begin{array}{l}\text { Émission neutronique du } \\
\text { combustible (fissions } \\
\text { spontanées + réaction } \\
(\alpha, n) n \mathrm{~s}^{-1} / \mathrm{t} \text { d'U) } \ldots .\end{array}$ & $2.10^{8}$ & $3,7 \cdot 10^{6}$ \\
\hline \multicolumn{3}{|c|}{ soit au total en curies par tonnes d'uranium : } \\
\hline & Eau légère & UNGG \\
\hline $\begin{array}{l}\Sigma \text { activité produits de } \\
\text { fission non gazeux.... } \\
\Sigma \text { activité produits de } \\
\text { fission gazeux....... } \\
\Sigma \text { activité } \alpha \ldots \ldots \ldots \ldots\end{array}$ & $\begin{array}{l}6,9 \cdot 10^{5} \\
9,5 \cdot 10^{3} \\
6800\end{array}$ & $\begin{array}{c}3,7.10^{5} \\
1,4.10^{3} \\
640\end{array}$ \\
\hline
\end{tabular}

- une activité des produits de fission non gazeux presque deux fois plus importante,

- une activité des produits de fission gazeux nettement plus élevée,

- une activité en tritium, qui se retrouvera sous forme d'eau tritiée, onze fois plus forte,

- une émission neutronique importante : à titre indicatif, l'émission neutronique du combustible lui-même provenant uniquement de fissions spontanées et de réactions $(\alpha, n)$, est cinquante fois plus forte, à masse égale;

On remarque en outre :

- un rapport des activités globales alpha/bêta cinq fois plus fort;

- un pourcentage d'uranium 235 résiduel dépassant celui de l'uranium naturel;

- une activité massique alpha du plutonium quatre fois plus importante dont l'essentiel provient de ${ }^{238} \mathrm{Pu}$.

\section{LES RISQUES ENGENDRÉS PAR LE FONCTIONNEMENT DE L'USINE}

\subsection{LeS RISQUES RADIOLOGIQUeS}

Nous sommes confrontés à La Hague à l'action de toutes les particules et rayonnements émis par les radionucléides décrits ci-dessus, ce qui nécessite la prise en compte, en pratique, des risques d'irradiation, des risques de contamination ou des deux risques simultanés.

vol. $15-\mathrm{N}^{0} 4$ 


\subsubsection{Les risques d'irradiation}

On distingue essentiellement :

- Les risques d'irradiation par les produits reçus.

Ils ne sont pas importants lors des opérations de réception et de déchargement en piscine des châteaux de transport, de même que durant le stockage en piscine à condition toutefois qu'il n'y ait pas corrosion du combustible, celle-ci se traduisant par une irradiation $\beta$ autour du plan d'eau.

Nous mentionnerons, néanmoins, le risque d'irradiation dû aux neutrons pour des combustibles transportés sous eau;

- Les risques d'irradiation par les produits traités.

Le risque d'irradiation par les photons existe à tous les stades du procédé. L'irradiation est due aux produits de fission jusqu'à la voie sèche plutonium, puis la contribution des photons issus des isotopes du plutonium, de l'uranium 237 et de l'américium 241 devient prépondérante lorsque l'activité résiduelle due aux produits de fission est négligeable, ce qui est le cas en pratique.

En exploitation normale, les écrans de protection ayant été largement dimensionnés, les débits de dose ambiants sont nettement inférieurs aux débits horaires limites habituellement envisagés pour les différentes zones concernées. Par contre, dans la partie haute de l'usine, il est fréquent de rencontrer des débits de dose de plusieurs dizaines de rad. $\mathrm{h}^{-1}$ lors d'interventions effectuées sur des circuits actifs, dans le cadre de la maintenance ou à la suite d'un incident, ce qui nécessite la mise en place de protections adéquates pour le personnel.

- Les risques d'irradiation par les produits livrés.

Les débits de dose issus d'une source d'oxyde de plutonium ne sont pas négligeables. En effet, pour du $\mathrm{PuO}_{2}$ provenant du retraitement de combustibles EL, les valeurs mesurées au contact d'un emballage contenant $3 \mathrm{~kg}$ de $\mathrm{PuO}_{2}$ sont de l'ordre de :

- emballage primaire : $1,5 \mathrm{rad} \cdot \mathrm{h}^{-1}$ dû aux photons;

- emballage complet : $450 \mathrm{mrad} \cdot \mathrm{h}^{-1}$ dû aux photons; $450 \mathrm{mrem} \cdot \mathrm{h}^{-1}$ dû aux neutrons.

- Les risques d'irradiation par les sous-produits.

Nous distinguons essentiellement : cation.

- Pour mémoire, les produits de fission stockés en cuve avant vitrifi-

- Les déchets solides de procédé et technologiques conditionnés suivant les spécifications techniques de l'Agence nationale pour la gestion des déchets radioactifs (ANDRA) $\left(<10 \mathrm{rad} . \mathrm{h}^{-1}\right.$ au contact).

- Les effluents liquides rejetés en mer qui ne sont pas irradiants.

- Les effluents gazeux, le radionucléide prépondérant étant ${ }^{85} \mathrm{Kr}$, qui entraînent une exposition de $30 \mathrm{mrem}$ à la peau, dans la zone de retombée 
maximale du panache de la cheminée, ceci pour 800 t de combustibles retraités.

\subsubsection{Les risques de contamination}

\section{Dans l'usine}

- Depuis la tête de l'usine jusqu'à la zone de moyenne activité, ce risque potentiel existe partout où sont véhiculées des matières radioactives, la nuisance essentielle étant celle des produits de fission.

- Dans les unités de récupération d'acide et d'eau, le risque est essentiellement dû au tritium et varie selon le recyclage de ces produits.

- Dans la zone de moyenne activité, l'on rencontre le risque de contamination par des produits d'activité spécifique très élevée comme le ${ }^{238} \mathrm{Pu}$ dont la contribution à l'activité $\alpha$ du plutonium est d'environ $75 \mathrm{p}$. cent pour du $\mathrm{PuO}_{2}$ provenant du retraitement du combustible EL irradié à $33000 \mathrm{MWj} / \mathrm{t}$.

\section{Dans l'environnement}

Les risques pris en compte concernent :

- l'environnement marin eu égard aux rejets liquides dont les activités maximales sont fixées par arrêté; les radionucléides considérés sont essentiellement le ruthénium ( 65 p. cent environ), le césium et le strontium ( 15 p. cent environ);

- l'environnement terrestre vis-à-vis des rejets gazeux et du passage possible de contamination dans le sous-sol du site.

\subsubsection{Le risque de criticité}

Bien que peu probable, ce risque est pris en compte dans certaines parties très limitées de l'usine.

\subsection{LES AUTRES RISQUES}

Il s'agit essentiellement :

- des risques de sécurité classique : incendie, explosion, inondation, manutention, risques chimiques, risques liés à l'utilisation d'appareils à pression de gaz et de fluides caloporteurs, au travail en hauteur, au travail en atmosphère bruyante...;

- d'une façon générale, des risques que peuvent présenter les installations classées pour la protection de l'environnement;

- des risques résultant de séismes ou de chutes de missiles.

\section{LE CONCEPT DE SÉCURITÉ DANS L'ÉTABLISSEMENT}

Les responsabilités en matière de sécurité s'exercent à deux niveaux : au niveau du directeur de l'établissement, au niveau des chefs d'installation. Pour assurer la sécurité des personnes et des biens de l'établissement, le directeur est assisté d'un ingénieur de sécurité d'établissement qui est en outre chef du Service de prévention et de radioprotection (S.P.R.). 
Le concept de sécurité retenu et mis en œuvre repose essentiellement sur des actions de prévention et sur des actions de surveillance et d'intervention.

\subsection{Les ACTIONS DE PRÉVENTION}

\subsubsection{Au niveau de l'installation}

\section{Lors de la conception de l'installation.}

Les règles générales suivant lesquelles sera prise en compte la sécurité de l'installation sont définies au départ. Elles concernent essentiellement :

- le dialogue entre le concepteur et l'exploitant pour tirer parti de l'expérience acquise;

- l'optimisation des protections à mettre en place entre un niveau d'irradiation en exploitation aussi faible que possible pour éviter les « doses gratuites » et le coût de celles-ci;

- la prise en compte des circuits des déchets radioactifs dans l'installation quel que soit le lieu de l'intervention ainsi que celle de "stockages tampon " éventuels;

- l'étude très complète des sas d'accès et de sortie de l'installation ainsi que celle de sas particuliers d'accès aux zones dans lesquelles on est susceptible d'intervenir fréquemment; ces études préalables visent essentiellement à éviter la dispersion de la contamination;

- le soin apporté à la ventilation des sous-ensembles de l'installation, notamment pour ce qui est de la filtration qui sera placée au plus près de la source, ceci pour supprimer l'irradiation des gaines, source de doses gratuites intégrées par le personnel;

- les modalités suivant lesquelles la maintenance sera effectuée, ceci pour la prise en compte, dès le départ, des emballages de protection des appareillages susceptibles d'être démontés et transportés vers l'atelier de décontamination; l'opération de maintenance devient ainsi analogue à une opération d'exploitation normale;

- enfin, un réseau de surveillance radiologique avec paramètres de fonctionnement, de préalarme et d'alarme, qui permette une bonne saisie de la situation et le déclenchement des moyens d'intervention dans les délais les meilleurs.

\section{Après mise en exploitation de l'installation.}

Bien que l'on essaie de tout prendre en compte lors de la conception, il convient, après la mise en exploitation, d'optimiser en quelque sorte la sécurité de l'installation par les actions suivantes :

- tout d'abord, il convient de dresser une carte radiologique de l'installation afin de vérifier que les hypothèses du projet sont bien respectées et que l'on ne met pas en évidence de fuites de rayonnements, ceci dans le cadre de la " chasse aux doses gratuites ";

- ensuite, à partir de ces résultats, compléter le travail du concepteur par l'étude fine in situ des différents postes de travail, c'est-à-dire l'étude du système " homme-environnement technologique et radiologique "; 
- enfin, des opérations ponctuelles de maintenance préventive retarderont, voire éviteront, des incidents radioactifs.

\subsubsection{Au niveau des personnels}

L'éducation des personnels constitue une action fondamentale de sécurité dans l'industrie nucléaire et, tout particulièrement, dans une usine de retraitement car un geste inutile ou une opération mal préparée augmentera inutilement le bilan radiologique de l'établissement.

Il convient donc de sensibiliser le personnel à la sécurité et ceci, avant même de lui délivrer un film dosimètre; c'est ainsi que deux cours de sensibilisation aux problèmes de sécurité sont dispensés sur l'établissement : l'un, de 3 jours, est obligatoire pour tout nouvel agent, qu'il soit COGEMA ou Entreprise; l'autre de 10 jours, s'adresse surtout aux chefs d'équipe ou aux techniciens exerçant des responsabilités de chantier en milieu hostile.

De plus, nous faisons largement appel aux techniques audiovisuelles pour des interventions banalisées (changements de ronds ou de panneaux de boîtes à gants, par exemple).

Il faut, enfin, signaler l'intérêt de la fiche de nuisance établie au moment de l'embauche et révisée chaque fois qu'un agent change de poste de travail, fiche à partir de laquelle le Service médical du travail (S.M.T.) va examiner l'aptitude de l'intéressé au poste de travail envisagé.

\subsection{LES ACTIONS DE SURVEILLANCE ET D'INTERVENTION}

Quelle que soit la qualité des hommes, du matériel et des techniques de radioprotection mises en œuvre, la radioprotection de l'établissement n'est efficace que dans la mesure où la meilleure collaboration règne entre l'exploitant et les services de sécurité (S.P.R., S.M.T., F.L.S. (*)). Il faut assister l'exploitant par le dialogue technique plutôt que par l'établissement de consignes draconiennes élaborées a priori.

\subsubsection{Les actions de surveillance en fonctionnement normal}

Nous mentionnerons, pour mémoire, l'existence des zones surveillées, des zones contrôlées et à l'intérieur de ces dernières, des zones à nuisances croissantes ventilées en conséquence.

Contrôle des installations.

Les types d'appareils sont choisis selon les risques décrits au paragraphe 3.1 ; ils doivent prendre en compte deux fonctions : la fonction alarme, la fonction dosimétrie ou, du moins, la meilleure approche possible de l'exposition subie par le personnel.

La fonction alarme est assurée par des détecteurs reliés à un tableau de contrôle de rayonnements; ces détecteurs ne peuvent assurer que l'information d'une variation du niveau du risque et, en aucun cas, une dosimétrie convenable pour le personnel qui se trouverait à proximité du lieu d'un

(*) F.L.S., Formation locale de sécurité.

VOL. $15-\mathrm{N}^{\circ} 4$ 
incident. Dans le cas de l'irradiation externe, la fonction dosimétrie est remplie par le port de dosimètres individuels; dans le cas de la contamination, elle l'est par la multiplication des prélèvements atmosphériques à comptage différé implantés à poste fixe ou portatifs, ainsi que par le contrôle systématique des personnels effectué par le Service médical du travail.

Jusqu'au premier cycle d'extraction ainsi qu'à la concentration et au stockage des produits de fission, la détection de la contamination est faite par la mesure $\beta \gamma$; après le premier cycle d'extraction, la détection de la contamination atmosphérique qui doit être effectuée sur le plutonium est compliquée par la contribution plus ou moins importante du ${ }^{238} \mathrm{Pu}$ dont la mesure implique la prise en compte d'un bruit de fond non négligeable.

\section{Contrôle du personnel.}

Le personnel est doté des dosimètres classiques : dosimètre à alarme, films dosimètres poitrine et poignet, stylo-dosimètre. L'appréciation des doses aux extrémités est faite à l'aide de bagues comportant des détecteurs thermoluminescents au LiF. Partout où existe un risque de criticité, le personnel est équipé de ceinture et de boîtier de criticité; par ailleurs, les équivalents de dose neutrons, compris entre 15 et 70 mrem sont mesurés tous les mois grâce aux dosimètres à albedo portés par le personnel, en double avec les plaques nucléaires impressionnées à partir d'un équivalent de dose de 100 mrem.

L'optimisation des postes de travail est obtenue d'une part, par l'exploitation mensuelle des résulstats dosimétriques, d'autre part, par l'examen des résultats des analyses systématiques d'urines et de selles effectuées par le S.M.T. et le L.A.M. (*).

\section{Contrôle des rejets gazeux.}

Les effluents gazeux rejetés par la cheminée et par les douze émissaires secondaires de l'usine sont contrôlés en continu; ce contrôle est classique et ne présente pas de difficulté particulière si ce n'est celle du contrôle instantané de ${ }^{131} \mathrm{I}$ en présence de ${ }^{85} \mathrm{Kr}$.

Le contrôle de l'environnement terrestre, marin et du sous-sol est basé pour l'essentiel, sur les prélèvements analysés aux laboratoires du S.P.R. La figure 2 illustre les modes de prélèvements effectués.

\section{Contrôle des rejets liquides.}

Les analyses réglementaires avant rejet sont effectuées par le S.P.R. pour que soient connues d'une part les activités spécifiques des radionucléides rejetés, d'autre part l'activité totale de l'effluent rejeté. Par délégation du directeur de l'établissement, le chef du S.P.R. donne ou refuse l'autorisation de rejet et vérifie que les activités rejetées restent dans les limites fixées. Un échantillon de chaque rejet est, par ailleurs, envoyé au Service central de protection contre les rayonnements ionisants (S.C.P.R.I.).

(*) L.A.M., Laboratoire d'analyses médicales. 


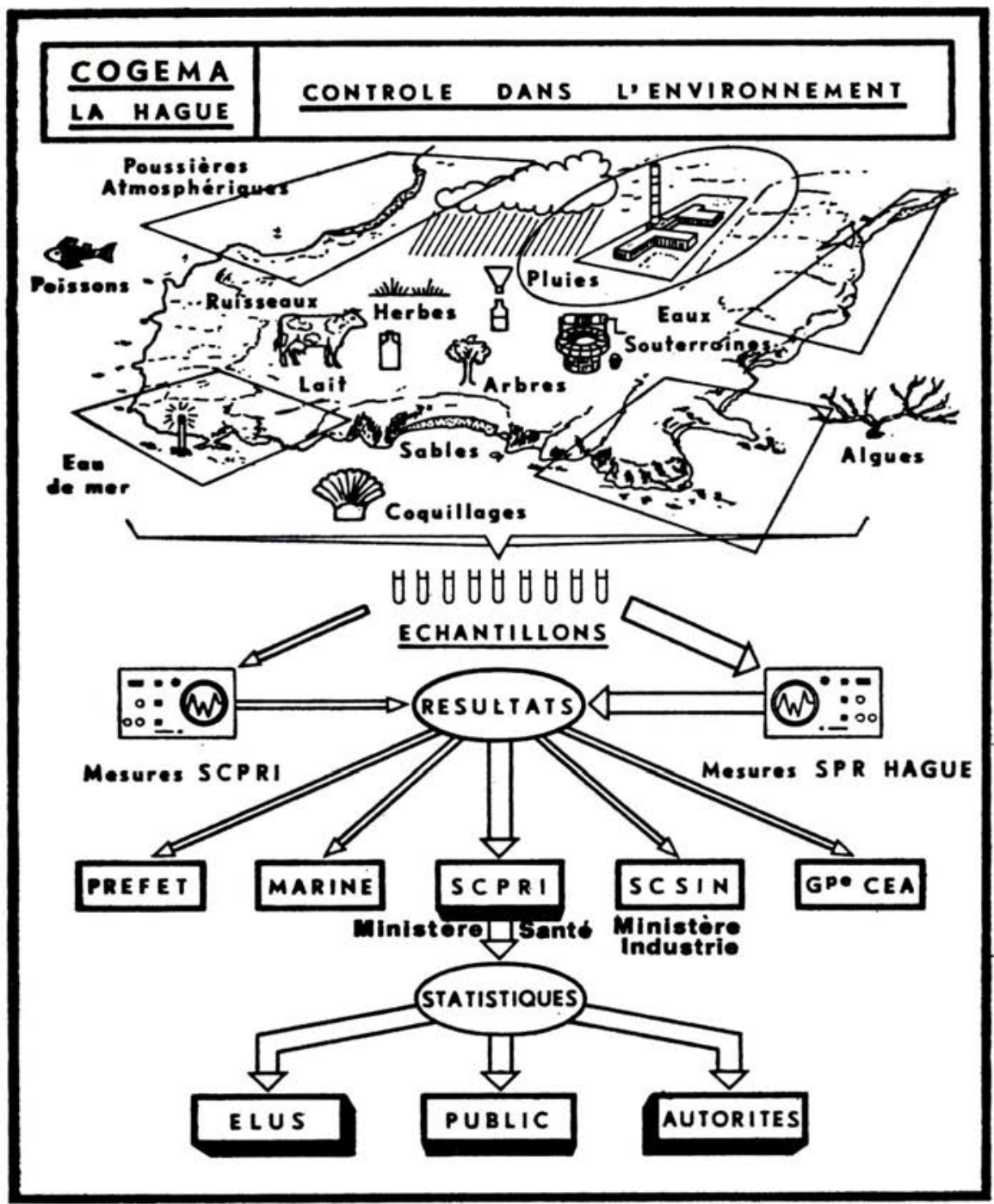

FIG. 2

Surveillance « criticité ».

La sûreté criticité est assurée par la géométrie chaque fois que le procédé le permet. Lorsque ce n'est pas le cas, des consignes d'exploitation permettent 
de limiter la masse ou la concentration de matière fissile. Enfin, des ensembles de détection " criticité " sont mis en place dans des zones particulières.

\subsubsection{Les actions de sécurité durant les interventions}

Il faut absolument que les conditions de travail durant les interventions soient adaptées aux risques réels. En effet, trop souvent les relations entre I e S.P.R. et l'intervenant se dégradent parce qu'une préparation insuffisante de l'intervention a entraîné la prescription de tenues de travail lourdes, inadaptées, donc contraignantes. Ce point est capital car, on le verra plus loin, c'est essentiellement durant les interventions, que le bilan radiologique de l'établissement s'alourdit.

Il convient donc d'étudier en détail les différentes phases de l'intervention avant son exécution de manière à optimiser les temps, de la même façon que l'on prépare l'exécution de travaux sous-marins pour lesquels le temps de séjour sous l'eau constitue le chemin critique. En effet :

- le travail en milieu hostile est contraignant par nature;

- il implique, de surcroît, l'isolement physique et psychologique de l'agent en intervention;

- bien que le débit de dose au poste de travail soit mesuré avant le début de l'intervention, il peut varier très sensiblement en cours d'exécution, la dose intégrée, elle, n'est connue qu'a posteriori;

- l'agent en intervention peut connaître des moments de faiblesse, voire de défaillance, il court un risque et peut le faire partager aux autres.

Dans ces conditions, tout homme a souvent un comportement différent de celui qu'il a habituellement. On ne peut donc lui demander, sinon la même qualité de travail, du moins la même efficacité.

Ces difficultés se traduisent par des coûts d'exploitation et d'intervention élevés. Pour réduire ces difficultés, leur coût et les durées d'intervention, nous effectuons, outre la préparation citée plus haut, un encadrement très serré des grosses interventions; cet encadrement s'appuie, dans certains cas, sur une assistance télédosimétrique et sur une assistance audiovisuelle. Un tel système visant à assister l'homme en milieu hostile contribue à améliorer les conditions de travail, à " sécuriser " et, par voie de conséquence, à augmenter l'efficacité des exécutants.

Lorsque la préparation et l'organisation du travail, compléments indispensables du système, sont bien établis, on obtient une réduction considérable de la durée des chantiers, de la dose intégrée globale, de la consommation de vêtements, de la consommation des tenues en PVC, de la production de déchets, du coût global des chantiers.

Par voie de retombées, l'on peut espérer accélérer l'initiation des néophytes au comportement en milieu hostile, améliorer la formation du personnel, diffuser vers les concepteurs d'installation des informations concrètes permettant de mieux comprendre les problèmes d'exploitation et de maintenance.

Enfin, un tel système bien utilisé par l'encadrement peut améliorer considérablement les méthodes de travail et les relations humaines. 


\section{BILAN ET PERSPECTIVES}

La stratégie poursuivie sur l'établissement de La Hague porte essentiellement sur les points suivants :

- un dialogue permanent entre le concepteur et l'exploitant afin de tirer parti de l'expérience acquise;

- un effort soutenu de sensibilisation des personnels sur l'importance des problèmes de sécurité et des " gestes " à accomplir suivant les cas; pour ce faire, les voies utilisées sont l'Unité de formation pratique, le Comité d'hygiène et de sécurité et le Comité spécial d'hygiène et de sécurité qui regroupe l'entreprise utilisatrice (COGEMA/LH) et les entreprises intervenantes de l'établissement;

- une appréciation permanente de l'équilibre à assurer entre la protection collective et la protection individuelle du personnel. En effet, un concept de sécurité basé sur une protection collective exagérée, outre le coût qu'il représente, créera un climat de sécurité factice, car il entraînera une démobilisation du personnel. Il faut donc étudier, cas par cas, le choix étant guidé par la recherche de l'optimisation entre la pénibilité du travail, les conditions du travail qui induiront la meilleure efficacité, et le coût d'aménagement du poste de travail;

- un dialogue permanent entre l'exploitant dans le sens le plus large et le personnel de soutien sécurité qui l'assiste. Ce " soutien sécurité 》 doit également être compris dans le sens le plus large, c'est-à-dire radioprotection, médical, sécurité incendie, sécurité du travail, criticité, etc. Tous les problèmes sont liés et leur complexité est telle que l'optimisation d'un poste de travail suppose que tous les spécialistes de l'établissement en matière de sécurité aient été consultés;

- une recherche régulière de l'appui des compétences du Service central de sûreté des installations nucléaires (S.C.S.I.N.) et du Service central de protection contre les rayonnements ionisants (S.C.P.R.I.).

\subsection{BILAN}

Bien que l'on alterne le retraitement de plusieurs types de combustibles, le bilan se présente favorablement :

- Les doses intégrées par le personnel durant les opérations d'exploitation sont relativement faibles.

- Nous appréhendons les problèmes de sécurité posés par les piscines de stockage renfermant des éléments fissurés. Cependant, il s'avère que les contrôles sérieux de combustibles effectués avant départ des centrales ainsi que les procédures actuelles de contact entre "l'électricien et le retraiteur " permettent d'éviter les surprises en piscines. En effet, nous nous orientons vers de grandes piscines; il faut donc une garantie totale de réception d'éléments sains. Deux cas pour 360 châteaux déchargés ont été détectés et ont fait l'objet d'une procédure particulière. Le traitement efficace des eaux et la bonne tenue des gaines permettent de maintenir l'activité de l'eau à quelques $10^{-5} \mathrm{Ci} / \mathrm{m}^{3}$.

vol. $15-\mathrm{N}^{\circ} 4$ 
- Nous maîtrisons bien les chantiers en milieu hostile en ne laissant aucune place à l'improvisation : nous savons protéger les personnels pour des travaux effectués dans des champs d'irradiation intense - plusieurs dizaines de rads par heure, parfois 200 à $500 \mathrm{rad} / \mathrm{h}$ - puisque les bilans radiologiques sont acceptables; parallèlement, le soin tout particulier apporté à la non-dispersion de la contamination ainsi qu'au contrôle du personnel en sortie de zone réduit à un niveau acceptable le nombre d'agents contaminés.

- Le matériel de protection et de contrôle individuels (protection des voies respiratoires et dosimétrie) dont sont équipés les personnels est bien adapté aux risques radiologiques rencontrés.

- Les résultats des mesures effectuées par le S.C.P.R.I. démontrent l'innocuité des activités de l'usine vis-à-vis de l'environnement; en effet, les activités contenues dans les effluents liquides rejetés ainsi que dans les effluents gazeux sont très inférieures aux limites autorisées jusqu'à ce jour.

- Le bilan radiologique du personnel pour 1979 est encourageant : 569 hommes-rems pour l'ensemble des personnels COGEMA + ENTRE-

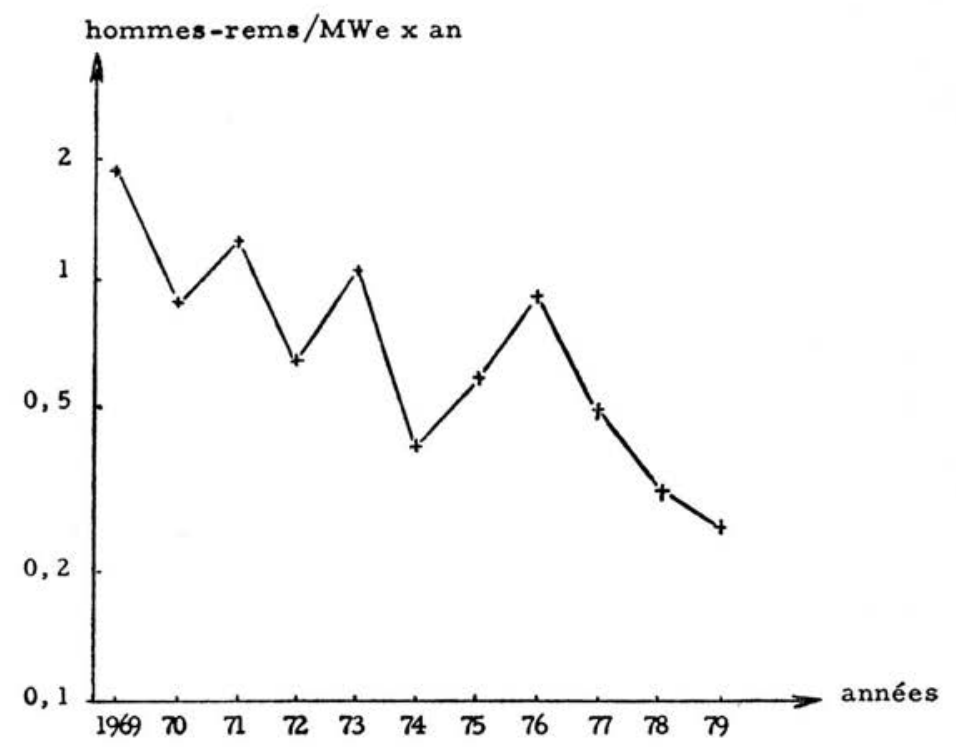

FIG. $3 a$. - Évolution des paramètres radiologiques 


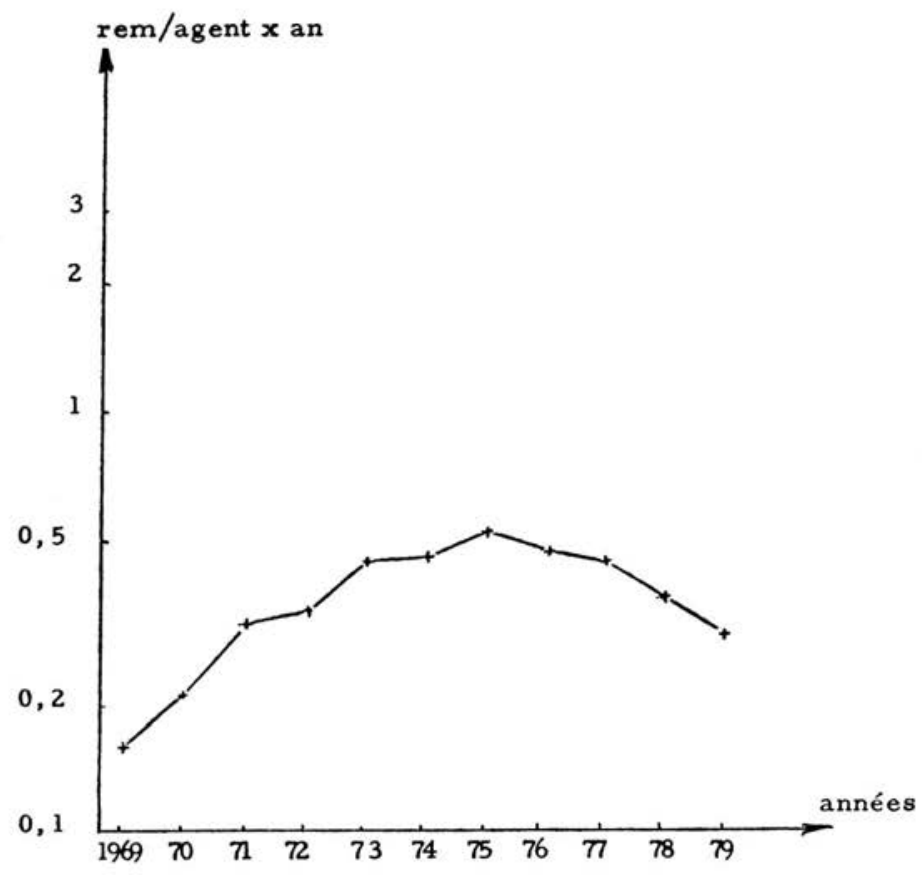

FIG. $3 b$. Évolution des paramètres radiologiques : équivalents de dose moyens annuels

PRISES exposés, soit 2000 personnes environ; la moyenne annuelle 1979 est de 297 mrem; de plus, 5 agents seulement ont des équivalents de dose compris entre 2,5 et 3 rem et aucun équivalent de dose n'est supérieur à 3 rem. Les figures $3 a$ et $3 b$ illustrent l'évolution des hommes-rems/MWe.an et des équivalents de dose moyens annuels de l'ensemble du personnel COGEMA + ENTREPRISES, de 1969 à 1979; on constate qu'en 1979, l'irradiation moyenne par agent a encore diminué par rapport aux années précédentes pour revenir au même niveau qu'en 1971; en effet, le facteur de diminution par rapport à l'année 1975, au cours de laquelle ont été intégrées les doses les plus importantes, est de 1,7. Si l'on compare les résultats des années 1979 et 1971 , on note que les combustibles traités représentaient respectivement $2544000 \mathrm{MWj}$ et $292000 \mathrm{MWj}$, soit une augmentation d'un facteur 8,7; exprimée en hommes-rems/MWe.an, l'évolution est de 1,3 en 1971 pour 0,26 en 1979 , soit une diminution d'un facteur 5 .

VoL. $15-\mathrm{N}^{\circ} 4$ 


\subsection{PERSPECTIVES}

Nous avons tout lieu de penser que le niveau d'optimisation des doses n'est pas encore atteint et que l'évolution favorable constatée depuis 1975 devrait se poursuivre dans le même sens en 1980 comme l'indiquent les résultats de dosimétrie des premiers mois de l'année. Nous pensons, néanmoins, que la portée des actions de sécurité décrites tout au long de ce rapport, s'avèrera limitée dans le temps.

Une autre approche pour réduire les doses consisterait à diminuer la fréquence des interventions; en effet, nous disposons à présent d'une formule semi-empirique d'estimation des intégrations par l' " organisme entier " liées aux activités de l'établissement et qui tient compte, notamment, de la production annuelle de plutonium ainsi que des interventions diverses en milieu nucléaire à partir de paramètres tels que le temps d'arrêt des installations et " l'âge " de ces installations, le terme " âge " ayant trait au vieillissement des matériels et des personnes mais aussi à la conception et au suivi des différentes opérations de décontamination et de réparations. L'application de cette formule montre, par exemple, que pour une année idéale au cours de laquelle le programme de retraitement serait intégralement respecté, le bilan radiologique COGEMA + ENTREPRISES serait alors 1,5 fois plus faible que dans l'hypothèse où ce programme ne serait pas respecté du fait de fréquentes interruptions de fonctionnement.

Ce ne sont donc pas les journées de production qui alourdissent le bilan radiologique d'une usine de retraitement, ce sont les journées pendant lesquelles l'usine est arrêtée; c'est, en effet, durant ces périodes que se déroulent de multiples interventions et que le bilan s'alourdit. Nous pourrions donc diminuer le nombre d'interventions en effectuant un certain nombre d'actions préventives; cette procédure devrait conduire à une augmentation sensible du nombre des journées de production et, de ce fait, à une réduction de la durée de l'intercampagne annuelle. 